\title{
Quantitative Analysis of Geometric-Pattern Features of Interstitial Infiltrates in Digital Chest Radiographs: Preliminary Results
}

\author{
Shigehiko Katsuragawa, Kunio Doi, Heber MacMahon, Laurence Monnier-Cholley, Junji Morishita, and \\ Takayuki Ishida
}

\begin{abstract}
We are developing a computerized method for detection and characterization of interstitial diseases based on a quantitative analysis of geometric features of various infiltrate patterns in digital chest radiographs. In our approach, regions of interest (ROls) with $128 \times$ 128 matrix size $(22.4 \mathrm{~mm} \times 22.4 \mathrm{~mm})$ are automatically selected, covering peripheral lung regions. Next, nodular and linear opacities, which are the basic compo. nents of interstitial infiltrates, are identified from two processed images obtained by use of a multiple-level thresholding technique and a line enhancement filter, respectively. Finally, the total area of nodular opacities and the total length of linear opacities in each ROI are determined as measures of geometric pattern features. We have applied this computer analysis to 72 ROls with normal and abnormal patterns that were classified in advance by six chest radiologists. Prelimi. nary results indicate that the distribution of measures of geometric-pattern features correlate well with radi. ologists' classification. These early results are encouraging, and further evaluation hopes to establish that this computerized method might prove useful to radiologists in their assessment of interstitial diseases. Copyright 1996 by W.B. Saunders Company
\end{abstract}

KEY WORDS: computer-aided diagnosis, interstitial lung disease, chest radiography.

I NTERSTITIAL LUNG disease is defined as an abnormality of the interstitial compartments of the lung, which may be caused by infiltration by inflammatory or neoplastic cells or may be a consequence of the accumulation of fluid or proteinaceous material. ${ }^{1}$ The shape and size of the small opacities caused by interstitial infiltrates in chest radiographs are very important to radiologists in their differential diagnosis of interstitial diseases. However, evaluation of interstitial disease in chest radiographs is considered one of the most difficult problems in diagnostic radiology. One of the reasons is the variation among radiologists in the terms that they use to describe radiographic patterns of small opacities, which are not defined objectively. ${ }^{2}$ Therefore, it is likely that objective descriptions of the shape and size of small opacities caused by interstitial infiltrates will aid radiologists in their assessment of interstitial diseases.
For detection and characterization of interstitial diseases, we have developed an automated method of quantifying lung textures in terms of the rms variation and the first moment of the power spectrum of the lung texture patterns; these represent the magnitude and the coarseness (or fineness) of lung textures, respectively, as reported previously. ${ }^{3-14}$ These two texture measures have been very useful for distinguishing between normal lungs and abnormal lungs with interstitial disease. ${ }^{7,10,13}$ However, these texture measures have not been so effective for distinguishing subtle abnormal lung textures that involve various patterns; eg, the texture measures for some round opacities are almost identical to those for other irregular opacities. ${ }^{9}$ This result indicates the need for a new approach for the distinction of subtle texture patterns in chest images.

In this study, we devised a computerized method based on quantitative analysis of geometric-pattern features of various interstitial infiltrates, such as nodular, reticular, and reticulonodular patterns, in digital chest radiographs to detect and characterize these abnormalities.

\section{MATERIALS AND METHODS}

Overall scheme of geometric-pattem feature analysis. The overall scheme of our approach to the geometric-pattern feature analysis is described later. First, conventional posteroauterior chest radiographs that were obtained with Lanex Medium screens and OC film (Eastman Kodak, Rochester, NY) at $125 \mathrm{kVp}$ with a $12: 1$ grid, are digitized by use of a laser scanner with a $0.175 \mathrm{~mm}$ pixel size and 1,024 gray levels. The characteristic curve of the laser scanner, which indicates the relationship between the optical density and the pixel value (gray level), has been carefully maintained so that the optical density range from 0.2 to 2.8 is

From the Kurt Rossmann Laboratories for Radiologic Image Research, Department of Radiology, The University of Chicago, Illinois.

Supported by USPHS Grants CA24806 and CA62625.

Address repnint requests to Kunio Doi, PhD, Department of Radiology, The University of Chicago, 5841 S. Maryland Ave, Chicago, IL 60637.

Copyright 1996 by W.B. Saunders Company

0897-1889/96/0903-0007\$3.00/0 
related linearly to the pixel value in the range of 920 to 30 (ie, 0.003 optical density/pixel value). The approximate shape and locations of peripheral lung regions are identified from the detection of ribcage edges and the diaphragm, which are determined based on edge detection techniques with first and second derivative on chest radiographs. Then, approximately 40 regions of interest (ROIs) with $128 \times 128$ matrix size are automatically selected, covering peripheral lung regions as shown in Fig 1. The nonuniform background trend, which is caused by normal lung structure and the variation in chest wall thickness, is corrected by means of a two-dimensional surface-fitting technique ${ }^{3}$ with a 6 th-order polynomial surface, for isolation of the fluctuating patterns of the underlying lung texture. It is known that interstitial infiltrates in chest images are basically composed of nodular and linear opacities. ${ }^{15}$ Therefore, nodular and linear opacities of interstitial infiltrates are identified from two processed images, which are obtained by use of a multiple-level thresholding technique and a line enhancement filter, respectively. Finally, the total area of nodular opacities and the total length of linear opacities in each ROI are determined as measures of geometric pattern features.

Detection of nodular opacities. Candidates for nodular opacities are detected by application of a thresholding technique to a trend-corrected image. However, a binary image obtained with the thresholding technique includes nodular opacities caused by interstitial infiltrates and also unrelated opacities caused by rib edges and large pulmonary vessels. Therefore, nodular opacities must be distinguished from unrelated opacities. We examined, as measures, the circularity of opacities and the nonuniformity of the distribution of the edge gradient orientation in the opacities.

The effective diameter ${ }^{16}$ of an opacity is defined by the diameter of a circle having the same area as that of the

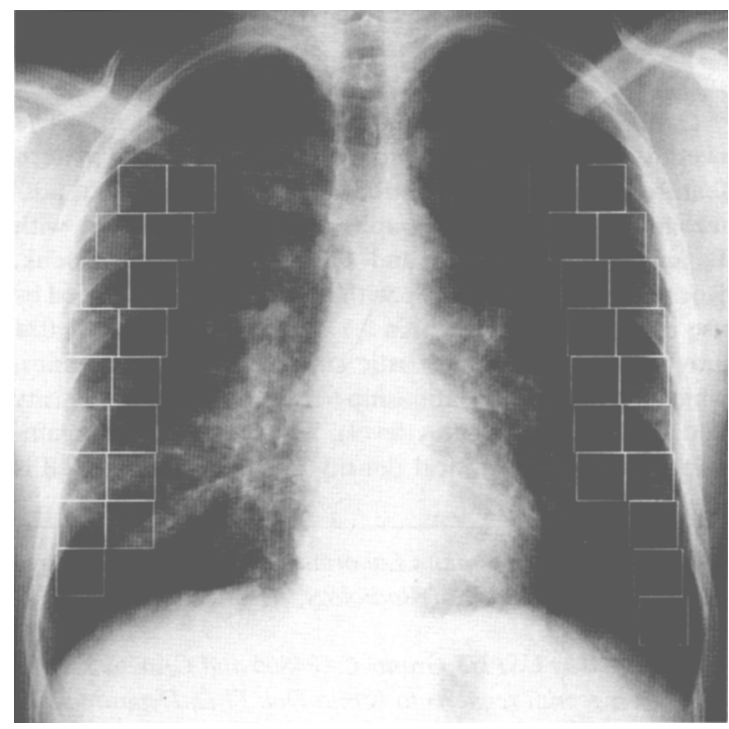

Fig 1. Illustration of the selection of ROls with a $128 \times 128$ matrix size for geometric-pattern feature analysis. opacity. The center of the equivalent circle is then located at the centroid of the opacity. The circularity is defined as the ratio of the area of the opacity within the equivalent circle to the actual area of the opacity. In general, the circularities for nodular opacities are larger than those for unrelated opacities such as rib edges and large vessels. However, it is difficult to distinguish nodular opacities from unrelated opacities based on a single threshold level, because the circularities of unrelated opacities change substantially, depending on the threshold level. For example, the circularities of rib edges tend to be smaller than those of nodular opacities at low threshold levels, but become large and almost comparable to those of nodular opacities at high threshold levels. Therefore, we employed a multiple-level thresholding technique to detect nodular opacities caused by interstitial infiltrates.

Five binary images for a trend-corrected ROI are obtained at five threshold levels that range from 5 pixel values to 25 pixel values, with increments of 5 pixel values. The threshold level is applied above the level of the background trend, which is estimated using the surface fitting technique as described earlier. The border details of each opacity in a binary image are removed by use of open and closed operations, which are basic morphologic filtering techniques. ${ }^{17}$ Next, the circularity of each opacity in binary images is monitored at various threshold levels. If the circularity of an opacity remains above 0.7 at a given threshold level and above it, then the opacity at the threshold ievel is identified as a nodular opacity. Opacities with small circularities (less than 0.7 ) at any threshold level are identified as unrelated opacities. However, some rib edges are occasionally divided into several segments with large circularities (above 0.7) at high threshold levels. Therefore, it is necessary to distinguish nodular opacities from segments of rib edges with large circularities at high threshold levels. We have found that the circularities of some nodular opacities are small at the threshold level of 5 pixel values because the threshold level is too low, and suddenly become large at higher threshold levels. On the other hand, rib edges generally retain small circularity up to the threshold level of 10 to 15 pixel values. Therefore, even though an opacity has a large circularity (above 0.7 ) at high threshold levels, it may be identified as an unrelated opacity if its circularity is smaller than 0.4 at the threshold level of 10 pixel values. It should be noted that the threshold circularities of 0.7 and 0.4 were determined empirically in this study. For optimization of the performance by this method, further studies will be needed to analyze a large number of cases.

Figures 2 a through e indicate opacities detected at threshold levels of $5,10,15,20$, and 25 pixel values, respectively. Figure $2 \mathrm{f}$ shows that nodular opacities can be distinguished from rib edges, where open (contours) and solid regions indicate nodular opacities and rib edges, respectively. The circularities for opacities $A, B$, and $C$ in Fig 2 a are plotted at each threshold level in Fig 3 . Because the circularity of opacity $A$ is larger than 0.7 at all of the threshold levels, this opacity is identified as a nodular opacity at the threshold level of 5 pixel values. Opacity B has a small circularity (below 0.7 ) at the threshold levels of 5 

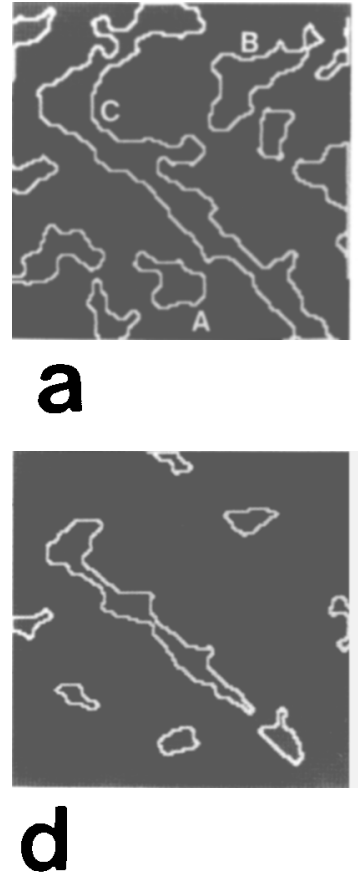

Fig 2. Demonstration of multiple-level thresholding for opacities detected at the threshold levels of 5 (a), 10 (b), 15 (c), 20 (d), and 25 pixel values (e). Nodular opacities (contours) can be distinguished from rib edges (solid regions) in (f).
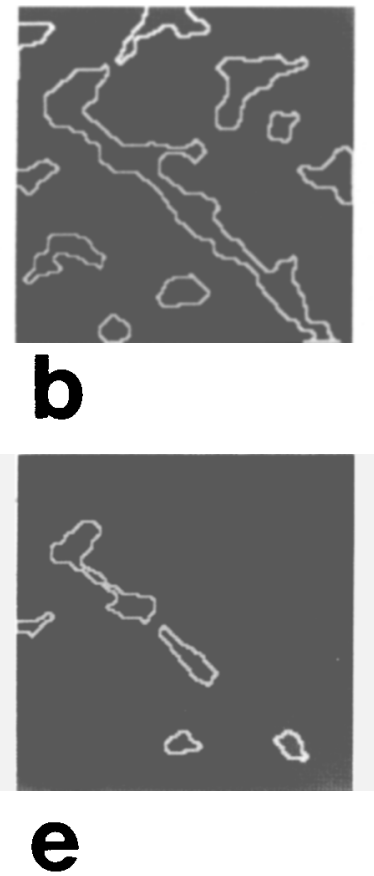

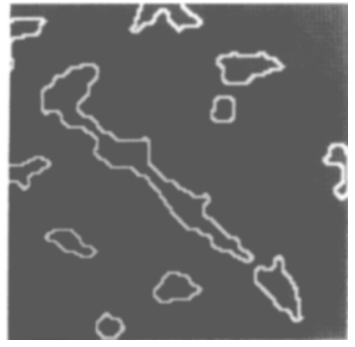

C

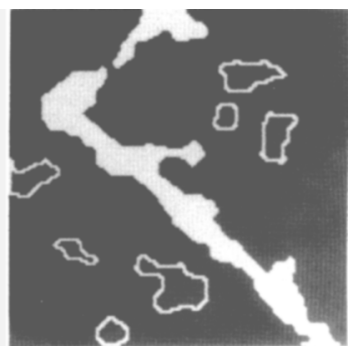

1

We used gradient-weighted edge orientation histogram analysis $^{12}$ in a further analysis for correct identification of nodular opacities. The edge gradient and its orientation are determined by use of the Sobel operator at each pixel within the opacity identified at the threshold level of 10 pixel values. Next, a gradient-weighted edge orientation histogram is obtained by accumulation of gradient values at each orientation interval of 30 degrees. Figure $4 \mathrm{~A}$ and $\mathrm{B}$ show a nodular opacity caused by an interstitial infiltrate and a rib edge, respectively. The corresponding gradient-weighted edge orientation histograms for the nodular opacity and the rib edge are shown in Fig $5 \mathrm{~A}$ and $\mathrm{B}$, respectively. It is obvious that the variation in the accumulated gradients of the histogram for the rib edge is much larger than that for the nodular opacity. The relative standard deviation of the accumulated gradients in the histogram, which corresponds to the standard deviation divided by the average of the gradients, indicates the magnitude of the variation in the histogram. Thus, the relative standard deviation is used as a measure for verifying the correct identification of nodular opacities; that is, opacities with less than 0.40 of the relative standard deviation are confirmed as nodular opacities caused by interstitial infiltrates.

Detection of linear opacities. Linear opacities caused by interstitial infiltrates are detected by application of a line enhancement filter to the trend-corrected ROI. The line enhancement filter is composed of eight templates, corresponding to eight directions for possible orientations of the lines to be detected, as shown in Fig 6. Each template is divided into three segments each of which consist of 12 pixels. These segments are located at a distance of one pixel

apart. The output value of a given template, $E_{i}(i=1$,

Fig 3. Relationship between circularity and threshold level for opacities A, B, and C shown in Fig 2 A. A and B are nodular opacities; $C$ is an unrelated opacity. 

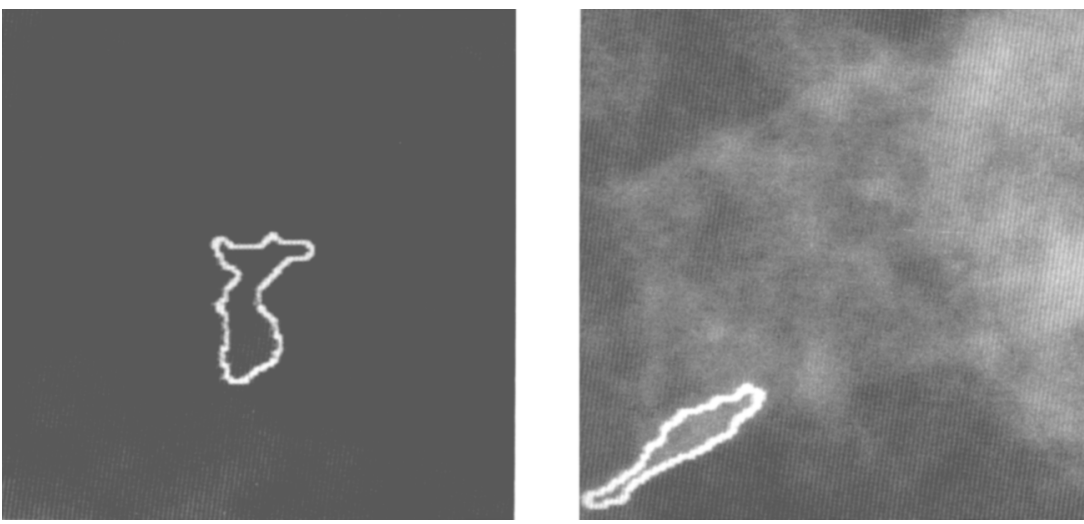

A

B
Fig 4. Illustration of a nodular opacity caused by an interstitial infiltrate $(A)$ and a rib edge $(B)$.
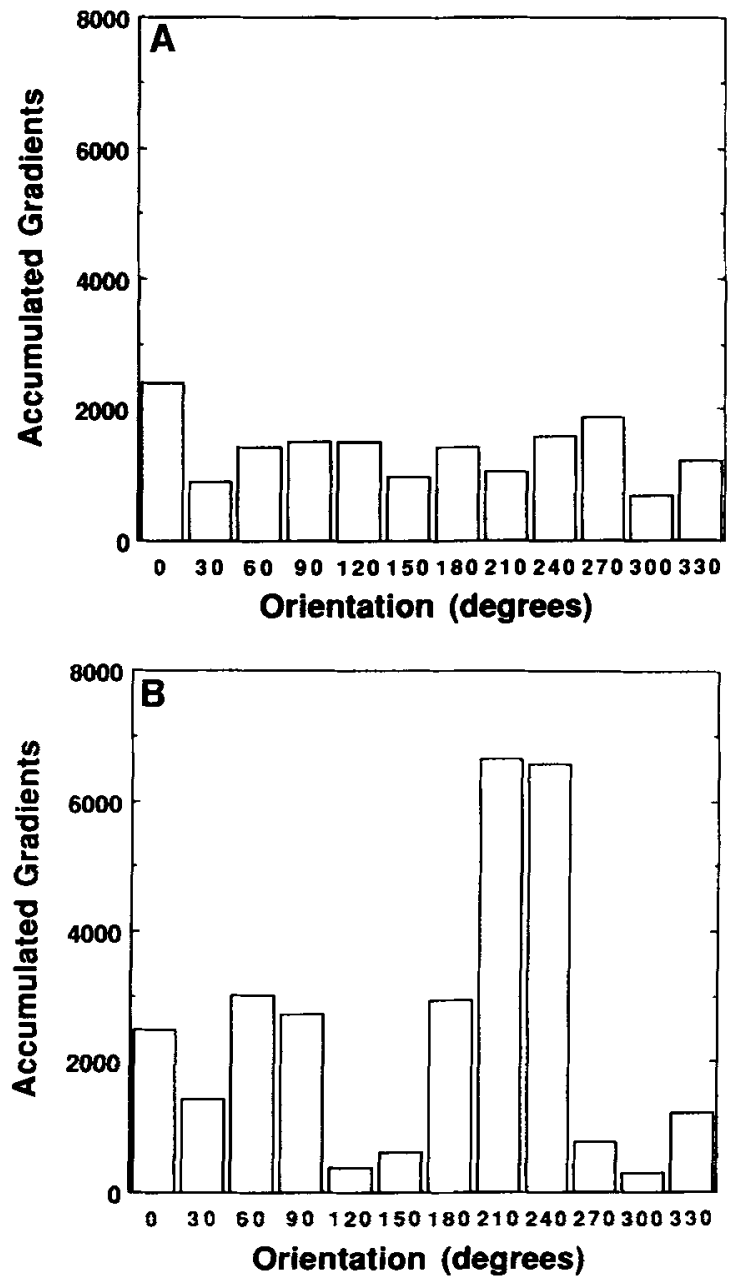

Fig 5. Gradient-weighted edge orientation histograms for the nodular opacity (A) and the rib edge (B) of Fig 4. The variation in the accumulated gradients for the rib edge is much larger than that for the nodular opacity.
$2, \ldots, 8$ ), is defined as follows:

$$
E_{i}=\left\{\begin{array}{ll}
2 B-A-C & (\text { if } B>A \text { and } B>C) \\
0 & \text { (otherwise) }
\end{array}\right. \text {, }
$$

where $\mathrm{A}, \mathrm{B}$, and $\mathrm{C}$ indicate the summation of pixel values in each segment of a template. The final output value, $E$, is determined, by processing with the line enhancement filter for a pixel in question, by the maximum value among the outputs of the eight templates as follows:

$$
E=\max \left\{E_{1}, E_{2}, E_{3}, E_{4}, E_{5}, E_{6}, E_{7}, E_{8}\right\}
$$

The line enhancement filter is applied to all pixels in an ROI. The low-contrast lines and short lines are then eliminated by thresholding regarding the output values of the filtered image and the length of the detected line pattern, respectively. Some lines identified by the line enhancement filter are overlapped with the nodular opacities or rib edges detected by the method previously described. This is because some nodular opacities tend to produce sharp line patterns along their edges. Therefore, linear opacities caused by interstitial infiltrates are finally identified by elimination of the line patterns that overlap with nodular opacities or rib edges.

Determination of measures for nodular and linear opacities. This computerized detection of nodular and linear opacities in an ROI is summarized in Fig $7 \mathrm{~A}$ through F. Figure $7 \mathrm{~A}$
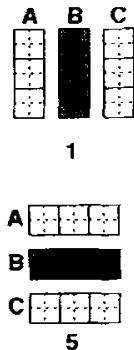

5

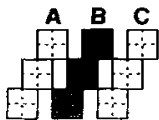

2

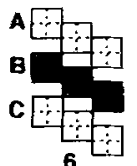

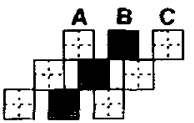

3

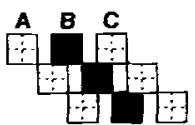

7
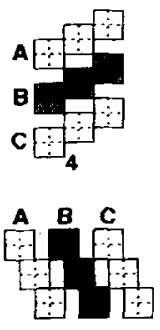

8
Fig 6. Line enhancement filter with eight templates corresponding to eight directions for possible orientations of the lines to be detected. 
Fig 7. Demonstration of nodular and linear opacities, including an original image with reticulonodular patterns (A); nodular opacities and rib edges identified by multiple-level thresholding technique (B); rib edges eliminated by use of the circularity and gradient-weighted edge orientation histogram analysis $(\mathrm{C})$; line patterns detected with the line enhancement filter (D); overlap of line patterns with nodular opacities or rib edges (E); and final detection of nodular and linear opacities after elimination of overlapping line patterns (F).

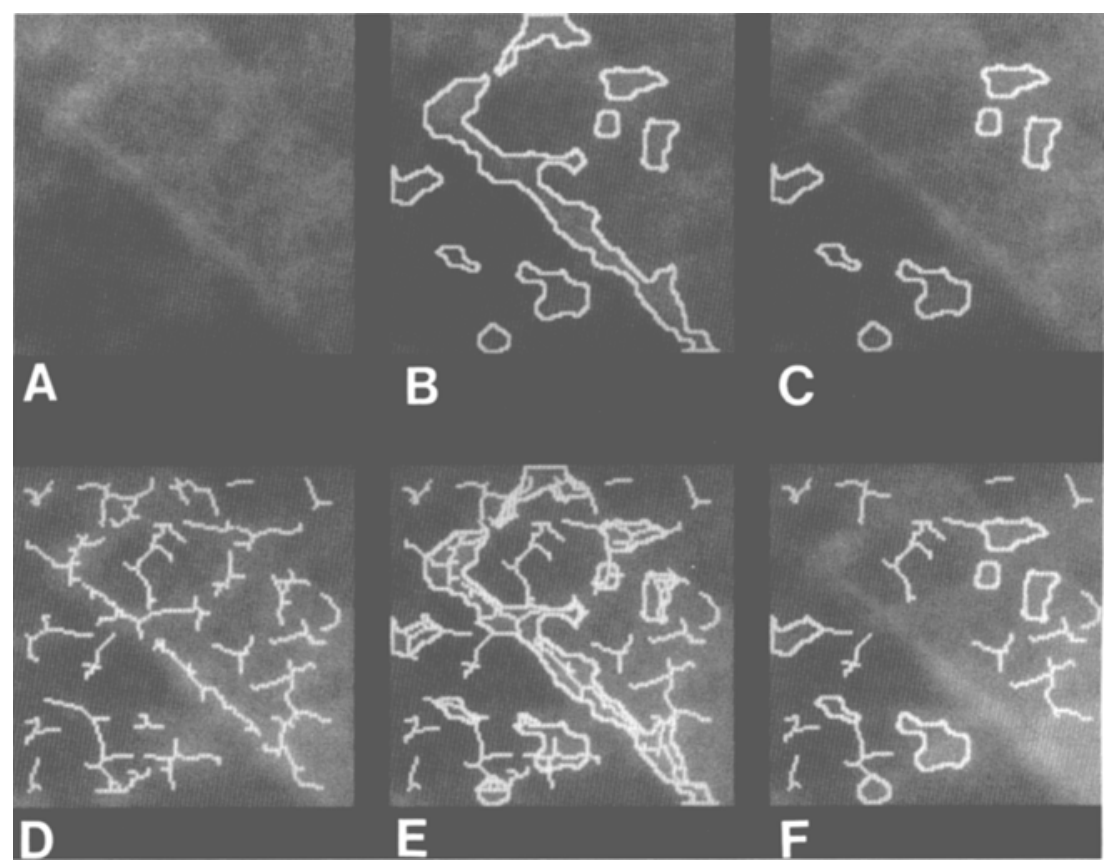

shows an original image with reticulonodular patterns. Figure 7 B shows contours of nodular opacities and rib edges obtained with the multiple-level thresholding technique. The rib edges are eliminated by analyses of the circularity and the nonuniformity of the gradient-weighted edge orientation histogram for the opacities, as illustrated in Fig $7 \mathrm{C}$. The line patterns are identified by use of the line enhancement filter, as shown in Fig 7D. Figure 7E illustrates an overlap of some line patterns with the nodular opacities or rib edges, which are detected in Fig 7B. Figure $7 F$ shows the final result for detected nodular and linear opacities caused by interstitial infiltrates after elimination of line patterns overlapped with nodular opacities and rib edges.

The total area of nodular opacities and the total length of linear opacities in an ROI are determined as measures of geometric pattern features for characterization of abnormalities of interstitial infiltrates. The total area of nodular opacities is defined by the ratio of the total number of pixels within all of the detected nodular opacities to the total number of pixels in the ROI. In addition, the total length of linear opacities is defined by the ratio of the total number of pixels, including all of the detected linear opacities, to the total number of pixels in the ROI.

\section{RESULTS}

We applied this computerized method for analysis of geometric-pattern features to some clinical cases. Figure $8 \mathrm{~A}$ through $\mathrm{D}$ illustrates portions of four chest images, including one normal and three abnormal lungs with nodular, reticular and reticulonodular patterns. Only one abnormal pattern is present in each of these three abnormal lungs. Only one ROI for each chest image was selected for this comparison. The corresponding measures of geometricpattern features are shown in Fig 9. It is apparent that the nodular pattern has a large total area of nodular opacities; the reticular pattern has a small total area of nodular opacities, and its total length of linear opacities is significantly larger than that of the normal lung;

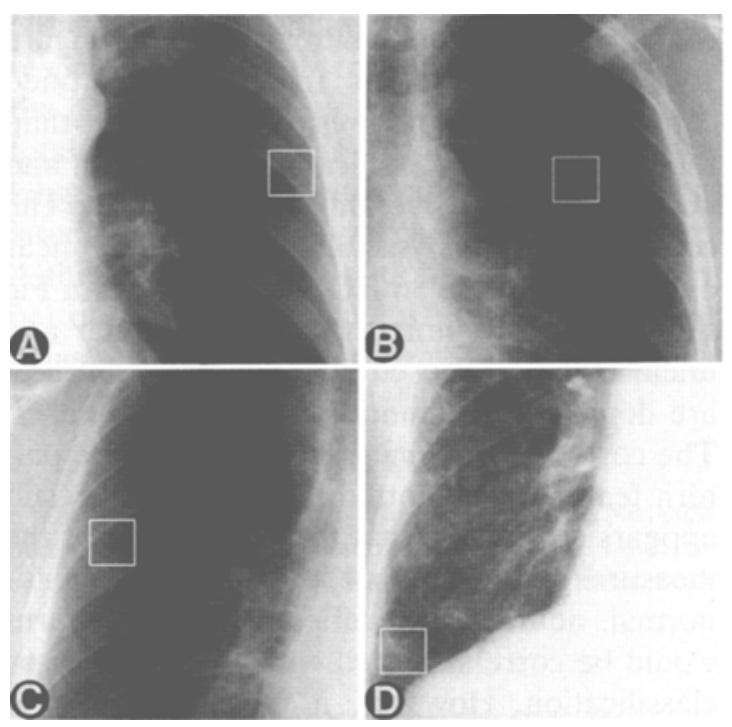

Fig 8. Portions of chest images of one normal lung (A) and of three abnormal lungs with nodular $(B)$, reticular $(C)$, and reticulonodular patterns (D). 


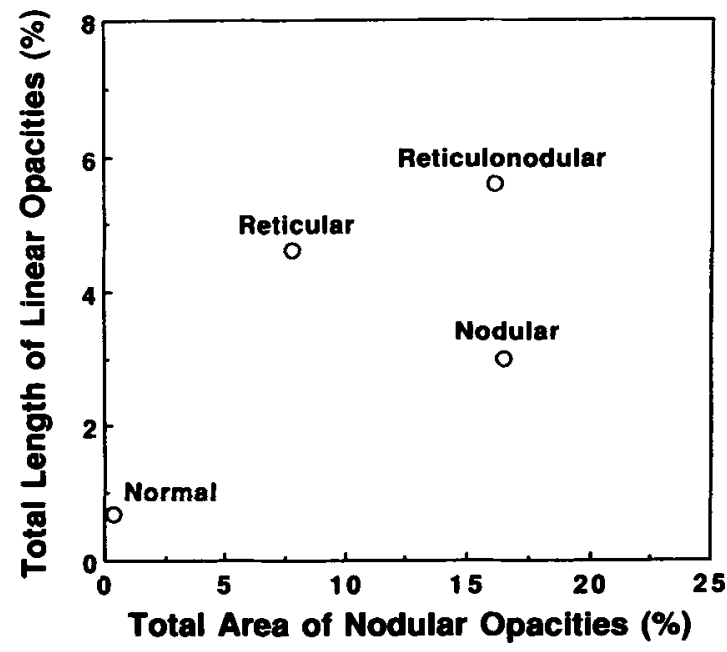

Fig 9. Distribution of two measures of geometric-pattern features for the selected ROls shown in Fig 8.

and the reticulonodular pattern has a large total area of nodular opacities and a large total length of linear opacities. These results appear to indicate that these two measures of geometricpattern features have the potential to distinguish three different types of abnormal patterns of interstitial infiltrates in chest images.

Six chest radiologists identified 72 ROIs including typical patterns from approximately 160 ROIs, which were selected automatically in the four chest images shown in Fig 8. Then, they classified those 72 ROIs into normal, nodular, reticular, and reticulonodular patterns. Figure 10 illustrates 36 ROIs that were identified consistently, with the consensus of more than four radiologists' classifications, as normal and abnormal with three different patterns. The computerized analysis of nodular and linear opacities for these 36 ROIs is illustrated in Fig 11. It should be noted that most nodular and linear opacities caused by interstitial infiltrates are detected by elimination of most rib edges. The corresponding measures of geometric pattern features are plotted in Fig 12. This result appears to indicate that the distribution of the measures for geometric pattern features for normal, nodular, and reticulonodular patterns would be correlated well with the radiologists' classification. However, it is very difficult to determine the distribution of measures for reticular patterns because only two ROIs with reticular patterns were available in this study.

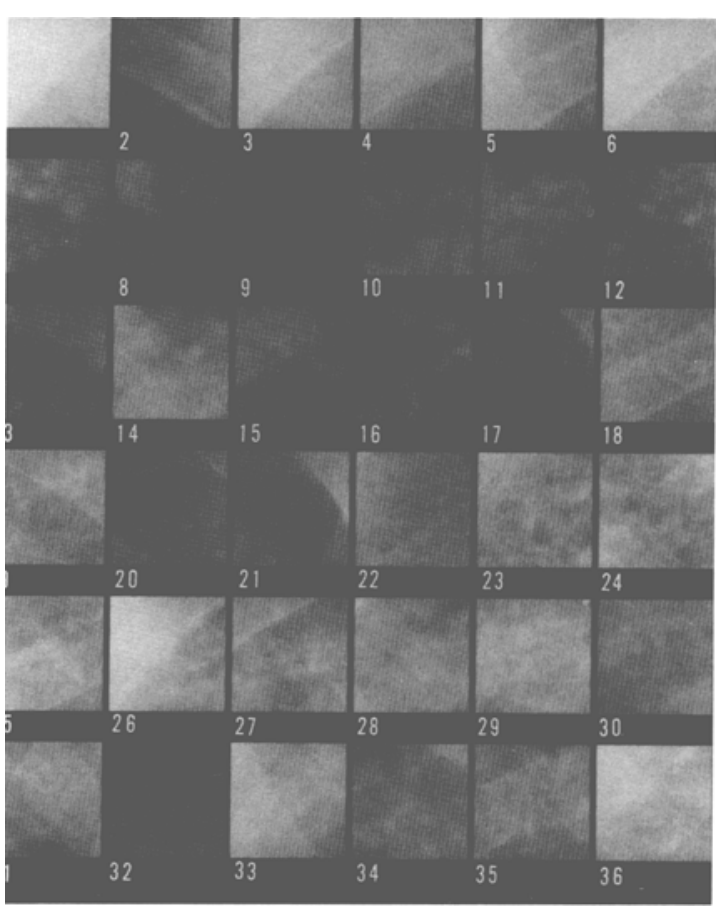

Fig 10. Illustration of 36 ROls with normal (1 to 6$)$ and abnormal patterns, including nodular (7 to 16), reticular (17 and 18 ), and reticulonodular (19 to 36 ) patterns, which were classified by radiologists.

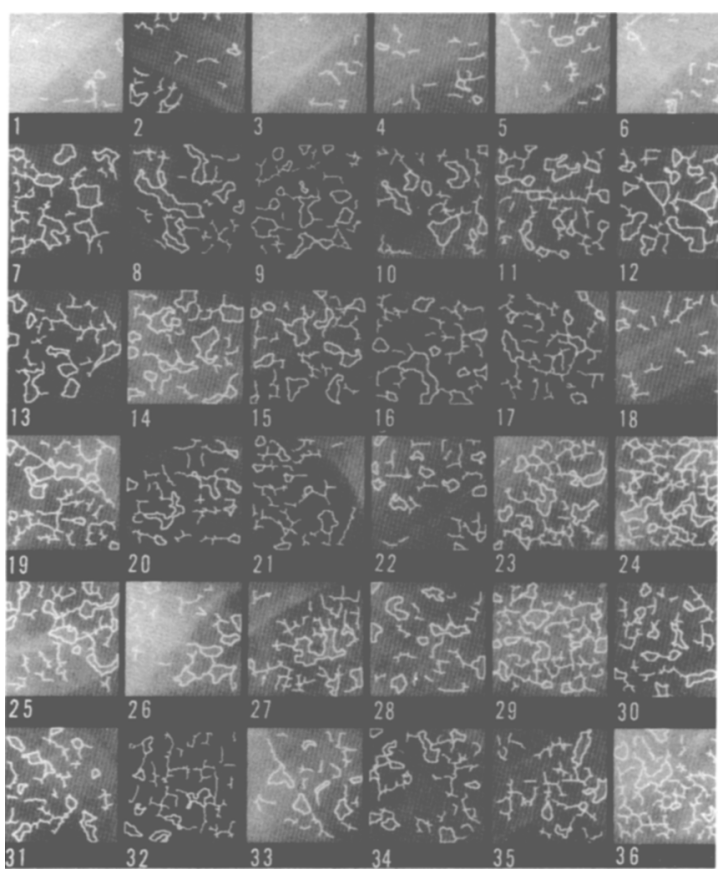

Fig 11. Detected nodular and linear opacities for the 36 ROls shown in Fig 10. 


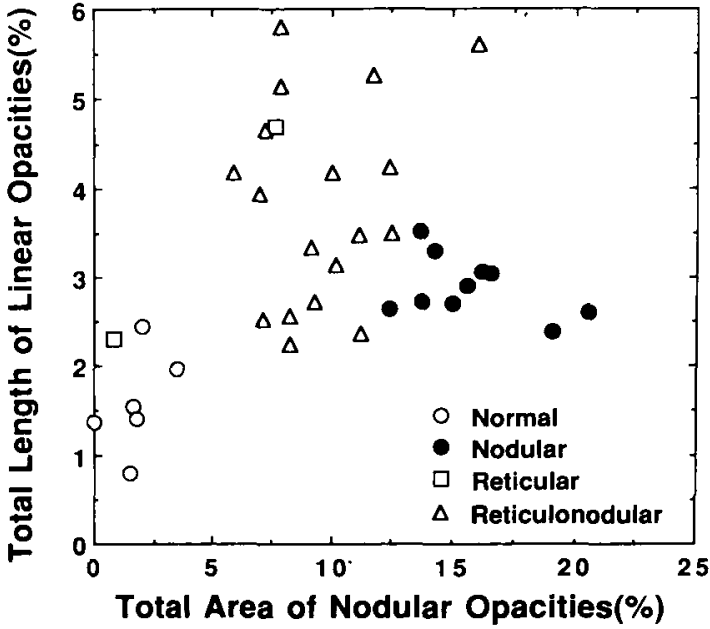

Fig 12. Distribution of two measures of geometric-pattern features for the 36 ROIs shown in Fig 10.

This is partly because predominantly reticular patterns are relatively unusual, such that only two ROIs were classified as reticular patterns with the consensus of four radiologists.

\section{DISCUSSION AND CONCLUSION}

We have developed a method for quantifying geometric-pattern features of interstitial infiltrates in digital chest radiographs to detect and characterize interstitial lung diseases. In this computerized method, the correction for a nonuniform background trend with a polynomial surface is very important as a preprocessing step for subsequent analysis. The order of the polynomial surface of the background trend must be selected according to the size of an ROI. ${ }^{3}$ A low order of the polynomial surface can be employed for a small ROI because the gross anatomy of the lung does not change substantially within a small ROI. However, because the ROI size used in this study was $22.4 \mathrm{~mm} \times 22.4$ $\mathrm{mm}$, it is desirable to employ a higher-polynomial surface to fit the background trend successfully. Therefore, we empirically chose a 6thorder polynomial surface for the background trend correction, based on visual comparison of the fitted polynomial surfaces with the trend- corrected images. However, further studies will be needed to examine whether a 6th-order or other polynomial surface may be optimal.

The rms variation and the first moment of the power spectrum used in the texture analysis ${ }^{3,12}$ can represent general features of nodular and linear opacities. However, as reported previously in the study for International Labor Organization standard chest radiographs of pneumoconioses, ${ }^{9}$ these texture measures of nodular opacities are similar to those of linear opacities when the diameter of the nodular opacities is comparable to the width of the linear opacities. This is probably because the power spectrum obtained from an ROI consisting of a number of nodular opacities is very similar to that for a number of linear opacities, although the individual power spectrum of a "single" nodular opacity may be different from that of a "single" linear opacity. On the other hand, with our new method of geometric-pattern feature analysis we can detect nodular and linear opacities separately in an ROI, and then determine the total area of nodular opacities and the total length of linear opacities. Therefore, it is expected that the geometric feature analysis would be more effective in distinguishing between nodular and linear opacities than are texture measures based on the power spectrum.

In conclusion, nodular and linear opacities are detected by means of a multiple-level thresholding technique and a line enhancement filter, respectively. The distribution of measures of the geometric-pattern features in terms of the total area of nodular opacities and the total length of linear opacities appears to be correlated well with the classification by radiologists. Although further evaluation with a large number of cases is necessary, we believe that these measures may be useful to radiologists in their assessment of interstitial diseases.

\section{ACKNOWLEDGMENT}

The authors are grateful to Xin-Wei Xu for his participation in elaborating the computer scheme, E. Lanzl for editing the report, and E.A. Ruzich for her secretarial work.

\section{REFERENCES}

1. Genereux GP: Pattern recognition in diffuse lung disease. Med Radiogr Photogr 61:2-31, 1985

2. Fraser RG, Pare JA: Diagnosis of Diseases of the Chest. Philadelphia, PA, Saunders, 1970
3. Katsuragawa S, Doi K, MacMahon H: Image feature analysis and computer-aided diagnosis in digital radiography: Detection and characterization of interstitial lung disease in digital chest radiographs. Med Phys 15:311-319, 1988 
4. Powell GF, Doi K, Katsuragawa S: Localization of inter-rib spaces for lung texture analysis and computeraided diagnosis in digital chest images. Med Phys 15:581587,1988

5. Katsuragawa S, Doi K, MacMahon $\mathrm{H}$, et al: Quantitative analysis of lung textures for detection and characterization of interstitial diseases in digital chest radiographs. Proc of Chest Imag Conf 138-143, 1988

6. Katsuragawa S, Doi K, MacMahon H: Quantitative analysis of lung texture for computer-aided diagnosis of interstitial disease in digital chest radiographs. SPIE 914:371374,1988

7. Katsuragawa S, Doi K, MacMahon H: Image feature analysis and computer-aided diagnosis in digital radiography: Classification of normal and abnormal lungs with interstitial disease in chest images. Med Phys 16:38-44, 1989

8. Katsuragawa S, Doi $\mathrm{K}$, Nakamori $\mathrm{N}$, et al: Image feature analysis and computer-aided diagnosis in digital radiography: Effect of digital parameters on the accuracy of computerized analysis of interstitial disease in digital chest radiographs. Med Phys 17:72-78, 1990

9. Katsuragawa S, Doi K, MacMahon H, et al: Quantitative computer-aided analysis of lung texture in chest radiographs. RadioGraphics 10:257-269, 1990

10. MacMahon H, Doi K, Chan HP, et al: Computeraided diagnosis in chest radiology. $J$ Thorac Imaging 5:6776,1990
11. Doi K, Giger ML, MacMahon H, et al: Computeraided diagnosis (CAD): Development of automated schemes for quantitative analysis of radiographic images. Semin Ultrasound CT MR 13:140-152, 1992

12. Chen X, Doi K, Katsuragawa S, et al: Automated selection of regions of interest for quantitative analysis of lung textures in digital chest radiographs. Med Phys 20:975982,1993

13. Abe K, Doi K, MacMahon H, et al: Computer-aided diagnosis in chest radiography: Preliminary experience. Invest Radiol 28:987-993, 1993

14. Morishita J, Doi K, Katsuragawa S, et al: Computeraided diagnosis for interstitial infiltrates in chest radiographs: Analysis of optical-density dependence on texture measures. Med Phys 22:1515-1522, 1995

15. Kido S, Ikezoe J, Naito $H$, et al: An image analyzing system for interstitial lung abnormalities in chest radiography. Invest Radiol 29:172-177, 1994

16. Giger ML, Doi K, MacMahon H: Image feature analysis and computer-aided diagnosis in digital radiography: 3. Automated detection of nodules in peripheral lung fields. Med Phys 15:158-166, 1988

17. Yoshimura $H$, Giger ML, Doi K, et al: Computerized scheme for the detection of pulmonary nodules: A nonlinear filtering technique. Invest Radiol 27:124-129, 1992 